\title{
Distinct Representations and Theta Dynamics in Dorsal and Ventral Hippocampus
}

\author{
Sébastien Royer, ${ }^{1,2}$ Anton Sirota, ${ }^{1}$ Jagdish Patel, ${ }^{1}$ and György Buzsáki ${ }^{1,2}$ \\ ${ }^{1}$ Center for Molecular and Behavioral Neuroscience, Rutgers, The State University of New Jersey, Newark, New Jersey 07102 , and ${ }^{2}$ Howard Hughes Medical \\ Institute, Janelia Farm Research Campus, Ashburn, Virginia 20147
}

\begin{abstract}
Although anatomical, lesion, and imaging studies of the hippocampus indicate qualitatively different information processing along its septo-temporal axis, physiological mechanisms supporting such distinction are missing. We found fundamental differences between the dorsal (dCA3) and the ventral-most parts (vCA3) of the hippocampus in both environmental representation and temporal dynamics. Discrete place fields of dCA3 neurons evenly covered all parts of the testing environments. In contrast, vCA3 neurons (1) rarely showed continuous two-dimensional place fields, (2) differentiated open and closed arms of a radial maze, and (3) discharged similar firing patterns with respect to the goals, both on multiple arms of a radial maze and during opposite journeys in a zigzag maze. In addition, theta power and the fraction of theta-rhythmic neurons were substantially reduced in the ventral compared with dorsal hippocampus. We hypothesize that the spatial representation in the septo-temporal axis of the hippocampus is progressively decreased. This change is paralleled with a reduction of theta rhythm and an increased representation of nonspatial information.
\end{abstract}

\section{Introduction}

Although the hippocampus is generally viewed as a single cortical module (Amaral and Lavenex, 2007; Wittner et al., 2007) (but see Andersen et al., 1971), a number of studies suggest a distinct or gradually changing computation along its septo-temporal axis. In support of this hypothesis, distinct sets of cortical and subcortical inputs reach different levels of the axis. The septal (dorsal) and midtemporal thirds receive visuospatial inputs from the dorsal entorhinal cortex (Dolorfo and Amaral, 1998; Witter and Amaral, 2004), whereas the temporal third receives most hypothalamic and amygdalar afferents carrying emotional information (Risold and Swanson, 1996; Petrovich et al., 2001). Septal cholinergic afferents from the fornix innervate more strongly the septal and midtemporal parts, whereas serotoninergic and dopaminergic afferents traveling along the amygdalo-hippocampal projection provide their strongest innervation to the temporal third (Gage et al., 1983; Verney et al., 1985; Witter et al., 1989; Pitkanen et al., 2000). Likewise, the septal and temporal portions of the hippocampus broadcast to different streams of structures (Van Hoesen and Pandya, 1975; Verwer et al., 1997; Amaral and Lavenex, 2007) and express differences in internal organization (Li et al., 1994), synaptic plasticity (Maggio and Segal, 2007), and molecular markers (Lein et al., 2007). In addition, diseases of the hippocampus affect different parts of the hippocampus. Seizures preferentially emanate from the uncal part of the

Received Sept. 20, 2009; revised 0ct. 27, 2009; accepted Nov. 5, 2009

This work was supported by National Institutes of Health Grants NS34994 and MH54671, and The James S. McDonnell Foundation. We thank K. Diba, E. Pastalkova, D. Sullivan, K. Mizuseki, and S. Fujisawa for comments.

Correspondence should be addressed to György Buzsáki, Center for Molecular and Behavioral Neuroscience, Rutgers University, 197 University Avenue, Newark, NJ 07102. E-mail: buzsaki@axon.rutgers.edu.

DOI:10.1523/JNEUROSCI.4681-09.2010

Copyright $\odot 2010$ the authors $\quad 0270-6474 / 10 / 301777-11 \$ 15.00 / 0$ human hippocampus and the analogous temporal third of the rodent hippocampus (Lieb et al., 1981; Bragdon et al., 1986). In contrast, ischemia selectively damages dorsal CA1 neurons in the rodent and the analogous CA1 region in the posterior (tail) part in humans (Ashton et al., 1989; Rempel-Clower et al., 1996). Finally, lesion and imaging experiments also suggest that the septal and temporal parts of the hippocampus support qualitatively different behaviors (Moser et al., 1995; Moser and Moser, 1998; Kjelstrup et al., 2002; Small, 2002; Bannerman et al., 2003; Bast et al., 2009).

In contrast to the anatomical, lesion, and clinical observations, physiological data supporting distinct representations along the septo-temporal axis are lacking. A large body of reports supports the role of the septal and midtemporal thirds of the hippocampus in spatial navigation (O'Keefe and Nadel, 1978; McNaughton et al., 2006). "Place cells" at the septal portion have discrete receptive fields (O'Keefe and Nadel, 1978; McNaughton et al., 2006), and the field size increases when recordings are made from the midtemporal (Jung et al., 1994) (but see Poucet et al., 1994; Maurer et al., 2005) or ventral third (Kjelstrup et al., 2008). However, nonspatial neuronal correlates (Wible et al., 1986; Poucet et al., 1994; Deadwyler et al., 1996; Hampson et al., 1999; Wood et al., 2000; Ferbinteanu and Shapiro, 2003; Johnson and Redish, 2007; Pastalkova et al., 2008) have not yet been studied in the ventral hippocampus. To examine potential physiological differences in the septo-temporal axis, we recorded local field potentials and unit firing in the septal and temporal thirds of the hippocampus in rats foraging in different testing environments.

\section{Materials and Methods}

Animals and surgery

Six Long-Evans rats (males; 250-400 g) were housed individually in transparent Plexiglas cages. Details of surgery and recovery procedures 
A

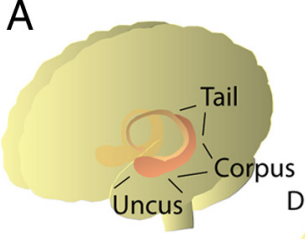

B

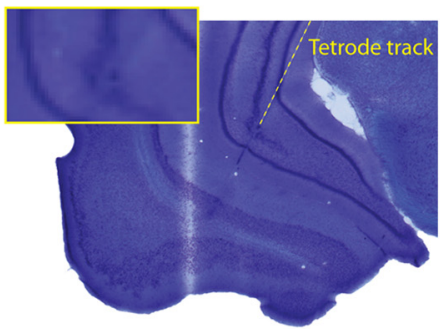

C

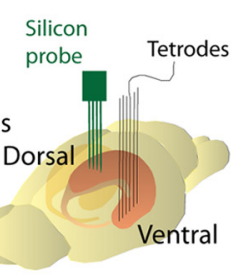

Ventral
Open arena

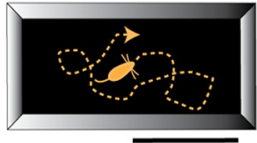

$1 \mathrm{~m}$

\section{Radial maze}

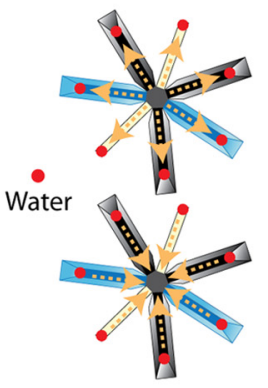

E Zigzag maze

Local field

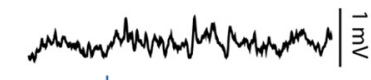

Units
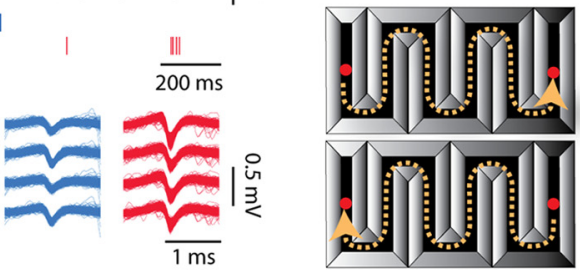

d CA3
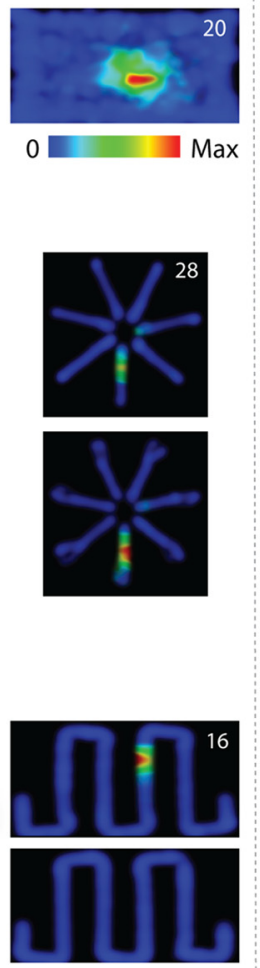

v CA3
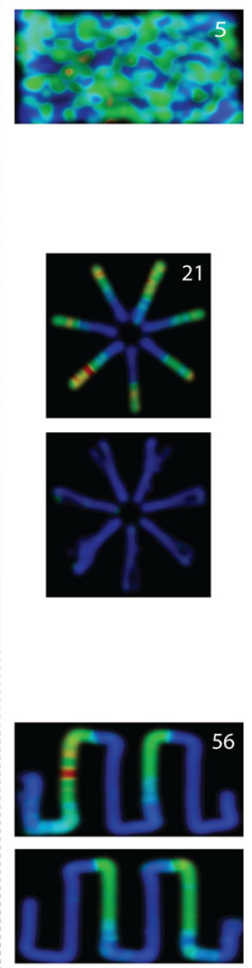

Figure 1. Experimental setup. $\boldsymbol{A}$, Regions of the hippocampus in the human and rat brains, and position of recording electrodes. $\boldsymbol{B}$, Histological illustration of the tetrode track and electrocoagulation lesion of the tip (inset) in the vCA3 pyramidal layer. Below, Example wideband ( $1 \mathrm{~Hz}$ to $8 \mathrm{kHz}$ ) trace of LFP recorded from the site shown above, raster plots of spiking for two neurons, and their spike waveforms (100 traces superimposed) on each channel of the tetrode. C, Left, The open arena had 30 -cm-high walls (in gray) and a $1 \times 2 \mathrm{~m}$ floor (in black). The dotted line shows the travel path of the rat. Center, Color-coded plot of firing rate as a function of rat's position for a representative neuron from dCA3. The number in the top right corner indicates the peak firing rate value corresponding to the red pixels. Right, Same display for a representative neuron from vCA3. D, Left, The seven-arm radial maze had two open arms and five arms with 35 -cm-high blue ( 2 arms) and black ( 3 arms) walls. The rat's trajectory has been decomposed into outbound (top) and inbound (bottom) travels for the analysis. Center, Representative neuron from dCA3 during outbound (top) and inbound (bottom) travels. Right, Same display for a representative neuron from vCA3. E, Zigzag maze. Left, The open arena could be converted into a zigzag maze by adding 30-cm-high partitions. The rat trajectory has been decomposed into rightward (top) and leftward (bottom) travels for the analysis. Center, Representative neuron from dCA3 during rightward (top) and leftward (bottom) travels. Right, Same display for a representative neuron from vCA3.

have been described previously (Csicsvari et al., 1998). The animals were deeply anesthetized with isoflurane. Two types of electrodes were implanted for unit and local field potential (LFP) recording. Four rats had eight independently movable wire tetrodes aimed to record from the dorsal (one tetrode) and ventral parts of the hippocampus (seven tetrodes). The tetrodes were composed of $12.5 \mu \mathrm{m}$ nichrome wires. The electrode tips were gold-plated to reduce electrode impedances to $\sim 300 \mathrm{k} \Omega$ at $1 \mathrm{kHz}$. Tetrodes aimed for both dorsal and ventral hippocampus recordings were inserted $-4.0 \mathrm{~mm}$ anteroposterior from bregma, $2.0 \mathrm{~mm}$ mediolateral with a $170^{\circ}$ incidence angle, to reach 2.6 $\mathrm{mm}$ mediolateral at $2 \mathrm{~mm}$ depth (initial position for dorsal hippocampus) and $4.5 \mathrm{~mm}$ mediolateral at $8 \mathrm{~mm}$ depth (initial position for ventral hippocampus), respectively. In two rats, a high-density 32 -site silicon probe (four-shank "octrode"; $20 \mu \mathrm{m}$ vertical site spacing in each shank; NeuroNexus Technologies), attached to a movable microdrive, was implanted in the dorsal hippocampus (anteroposterior, $-4.0 \mathrm{~mm}$; mediolateral, $2.6 \mathrm{~mm}$ ), in addition to eight tetrodes in the ventral hippocampus. In all experiments, ground and reference screws were implanted in the bone above the cerebellum. Over the course of several days, the tetrodes and silicon probes were lowered in steps of $60 \mu \mathrm{m}$ until pyramidal cells with spike bursts were isolated at appropriate depths. After each recording session, the electrodes were moved further until new well separated cells were encountered. At the end of the physiological recordings, a small direct current ( $5 \mu \mathrm{A} ; 10 \mathrm{~s})$ was applied to selected sites and the rat was deeply anesthetized and perfused with a fixative. The position of the electrodes was confirmed histologically, using Nissl-stained coronal sections. All experiments were performed in accordance with protocols approved by the Rutgers University Animal Care and Use Committee.

\section{Behavioral training and testing}

The animals were trained for 2 weeks before surgery in the three mazes (open, radial, and zigzag). For the radial and zigzag maze experiments, the animals were deprived of water for $24 \mathrm{~h}$ before the tasks. The same behavioral procedures were used for training and testing. The testing apparatus was separated from the experimenter and recording equipment by a black curtain, which also served as a polarizing cue relative to the white walls of the room. The floor and walls of the mazes were washed between sessions.

Open maze. The animals were trained to forage for small pieces $(\sim 3 \times$ $3 \mathrm{~mm}$ ) of Froot Loops (cereal; Kelloggs) on the open maze $(1 \times 2 \mathrm{~m})$ (see Fig. $1 C$ ) thrown one at a time from behind a curtain. Subsequent pieces were thrown only after the previous one was found. The open maze had side walls of $30 \mathrm{~cm}$ high on all four sides, tilting outward by $60^{\circ}$. The rat could freely see distant room cues.

Radial maze. Figure $1 D$ shows the dimensions of the seven-arm radial maze. The walls of the five closed arms were $35 \mathrm{~cm}$ high and tilting outward by $60^{\circ}$. These walls limited but did not prevent the rats' vision of distant room cues. Three arms were painted black, and two, blue. The remaining two arms had no walls (open arms). The animals were trained to seek for water rewards at the end of each arm (marked by red dots in Fig. $1 D)$. An equal amount of water $(20 \mu \mathrm{l})$ was added in all water wells regularly ( every 30 s), so that the wells that have not been visited for the 
A

dCA3

$\begin{aligned} \text { \% active pixels: } & 3.3 \\ \text { Contour size (\%): } & 3.4 \\ \text { Spatial information: } & 3.6 \\ \text { Stability: } & 0.87\end{aligned}$
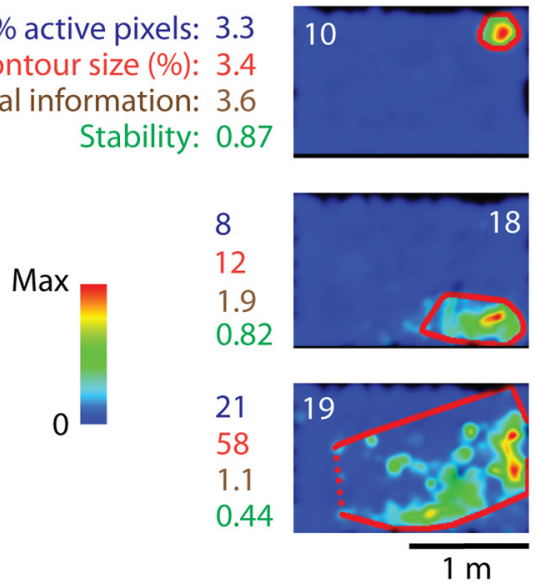

B

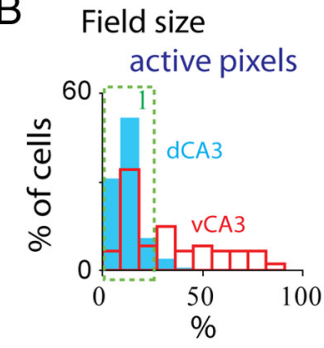

$\mathrm{D}$

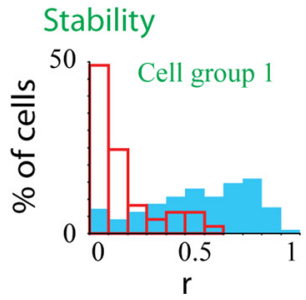

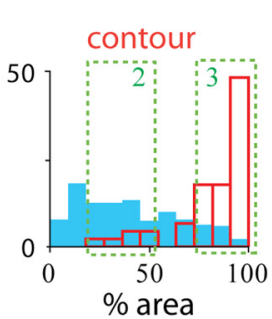

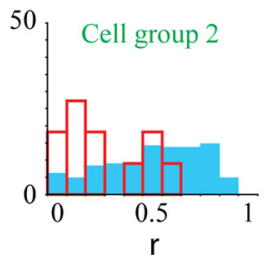

vCA3
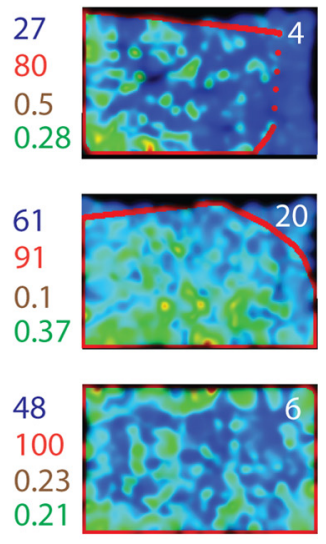

C Spatial information
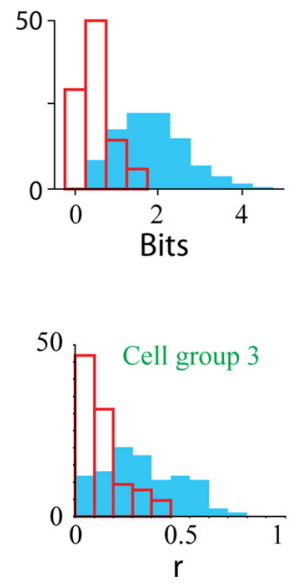

Figure 2. Firing fields on the open arena. $A$, Firing fields of representative pyramidal cells from the dCA3 and vCA3 regions. Firing rates are normalized to the peak (maximum) rate (white numbers) and color-coded. On the left of each example firing field are the corresponding values of firing field sizes (percentage of active pixels and firing field contour size), stability of activity between first and second halves of the recording session, and information content (Markus et al., 1994). B, Distributions of field sizes and contour sizes for all neurons. The distributions of dCA3 and vCA3 pyramidal cells are significantly different ( $p<0.01, t$ tests). $\boldsymbol{C}$, Distributions of information content for all neurons ( $p<0.01, t$ test). $\boldsymbol{D}$, Firing rate/pixel stability distributions are shown separately for subgroups of $\mathrm{dCA} 3$ and $\mathrm{vCA} 3$ neurons indicated by the boxes in $\boldsymbol{B}$. The rate/position stability of $\mathrm{vCA} 3$ pyramidal neurons was significantly lower than those of the dCA3 cells, regardless of the firing field size ( $p<0.01, t$ tests).

longest time accumulated more water. This approach ensured that the animals visited all arms with the same probability.

Zigzag maze. By placing five roof-shaped partitioning walls into the open maze, it was converted into a zigzag maze with 11 corridors (see Fig. $1 E$ ). The walls were $30 \mathrm{~cm}$ high with a $24 \mathrm{~cm}$ base and painted black. The animals learned to run back and forth between two water wells; $100 \mu \mathrm{l}$ of water was delivered at each well (see Fig. $1 E$, red dots).

Data acquisition, processing, and analysis

During the recording session, the rat was connected to a counterbalanced cable that allowed the animal to move freely in the apparatus. Wideband $(1 \mathrm{~Hz}$ to $8 \mathrm{kHz}$ ) extracellular electrical signals were preamplified $(20 \times)$ and digitized at $32 \mathrm{kHz}$ sampling rate at 24 bit resolution and stored for off-line analysis (DigiLynx system; NeuraLynx). Raw data were preprocessed using custom-developed suite of programs (Csicsvari et al., 1998). The wideband signal was downsampled to $1.25 \mathrm{kHz}$ to generate the LFP and was high-pass filtered $(>0.8 \mathrm{kHz})$ for spike detection.

Spike sorting and cell classification. Spike sorting was performed offline using a semiautomatic, custom-developed clustering analysis program (http://klustakwik.sourceforge.net) (Harris et al., 2000), followed by manual adjustment of unit clusters, aided by autocorrelation and

cross-correlation functions as additional separation tools (http://klusters.sourceforge.net; http://neuroscope.sourceforge.net) (Hazan et al., 2006). Only clusters with clear boundaries in at least one of the projections were included in the database (supplemental Fig. 1, available at www.jneurosci.org as supplemental material). Criteria for neuron type classification of unit clusters into putative pyramidal cells and interneurons are widely accepted in the dorsal hippocampus, based on firing rates, waveform features, and complex spike bursting patterns (Csicsvari et al., 1998). However, similar classification criteria in the ventral hippocampus are not yet available. In a previous study, pyramidal cells were distinguished from interneurons in the intermediate and ventral CA3 regions by the occurrence of complex spikes, spike width, and firing average rate (Kjelstrup et al., 2008). However, we found that complex spike bursts are less frequent in the ventral CA3 (vCA3) compared with the dorsal CA3 (dCA3) region (supplemental Fig. $2 C$, available at www.jneurosci.org as supplemental material). After testing various criteria, we found that the two parameters that allowed for the best separation between the two putative anatomical groups in the ventral CA3 region were firing rate and behavior-related firing pattern. Cells with a mean firing rate $>10 \mathrm{~Hz}$ per session and with firing field $>5 \mathrm{~Hz} /$ per pixel, covering $>80 \%$ of the maze area, were classified as putative interneurons. Other cells were classified as pyramidal cells (supplemental Fig. $2 \mathrm{~A}$, available at www.jneurosci.org as supplemental material). No attempt was made to distinguish among the large family of interneurons (Freund and Buzsáki, 1996). These initial separation criteria in the ventral hippocampus should be verified and improved in future experiments.

Two-dimensional firing field plots. For tracking the position of the animal, two small lightemitting diodes, mounted above the head stage, were recorded by a digital video camera at a rate of 30 samples per second. The open field and maze areas were divided into $200 \times$ 200 pixels. The number of spikes and occupancy times were calculated in each pixel to generate spike count and occupancy time matrices. Both matrices were smoothed by convolving them in two dimensions with a Gaussian function (5 pixels half-width). A ratio of "spike count/pixel occupancy" was plotted to generate "rate maps." The color of pixels corresponds to the firing rate of the cell, and the brightness corresponds to the occupancy time of the pixel. Only pixels with occupancy time $>200 \mathrm{~ms}$ are represented in the figures. For the zigzag maze plots, the ventral cells were smoothed using a larger Gaussian function (15 pixels half-width). This was done to compensate for the stronger sparseness of spikes caused by the larger fields of ventral cells.

Field sizes. The field sizes were defined by two complementary methods. In the first method, the number of pixels with firing rate values $>20 \%$ the cells peak rates were divided by the total number of pixels. This approach provides a precise number of "active" pixels, regardless of the spatial continuity of the firing fields. In the second method, the area within the shortest convex contour line, enclosing all active pixels, was divided by the total area.

Field stability. Since the firing rate map provides only a mean value for each pixel and does not distinguish whether the mean rate is attributable to a single or multiple visits, we also assessed field "stability." The field 

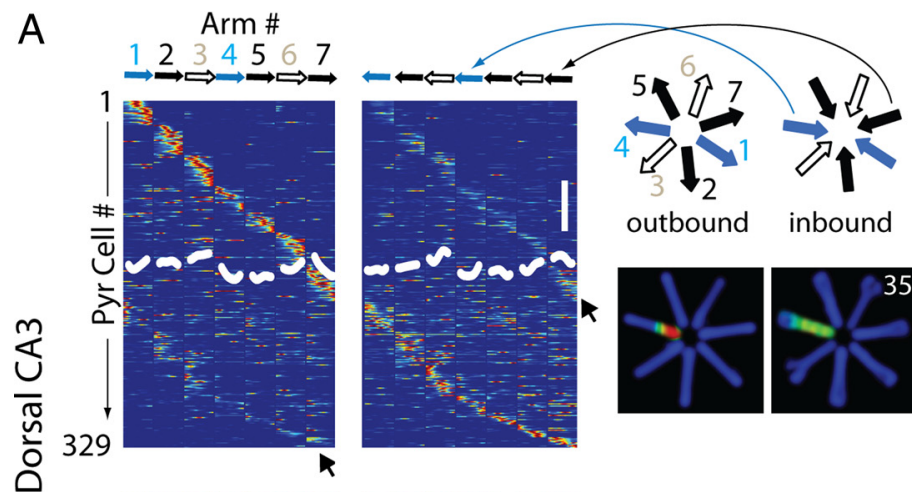

C

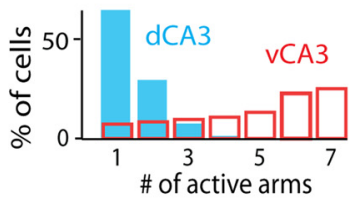

outbound inbound

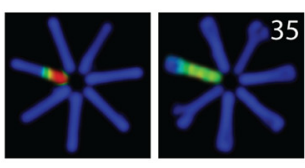

\section{D}
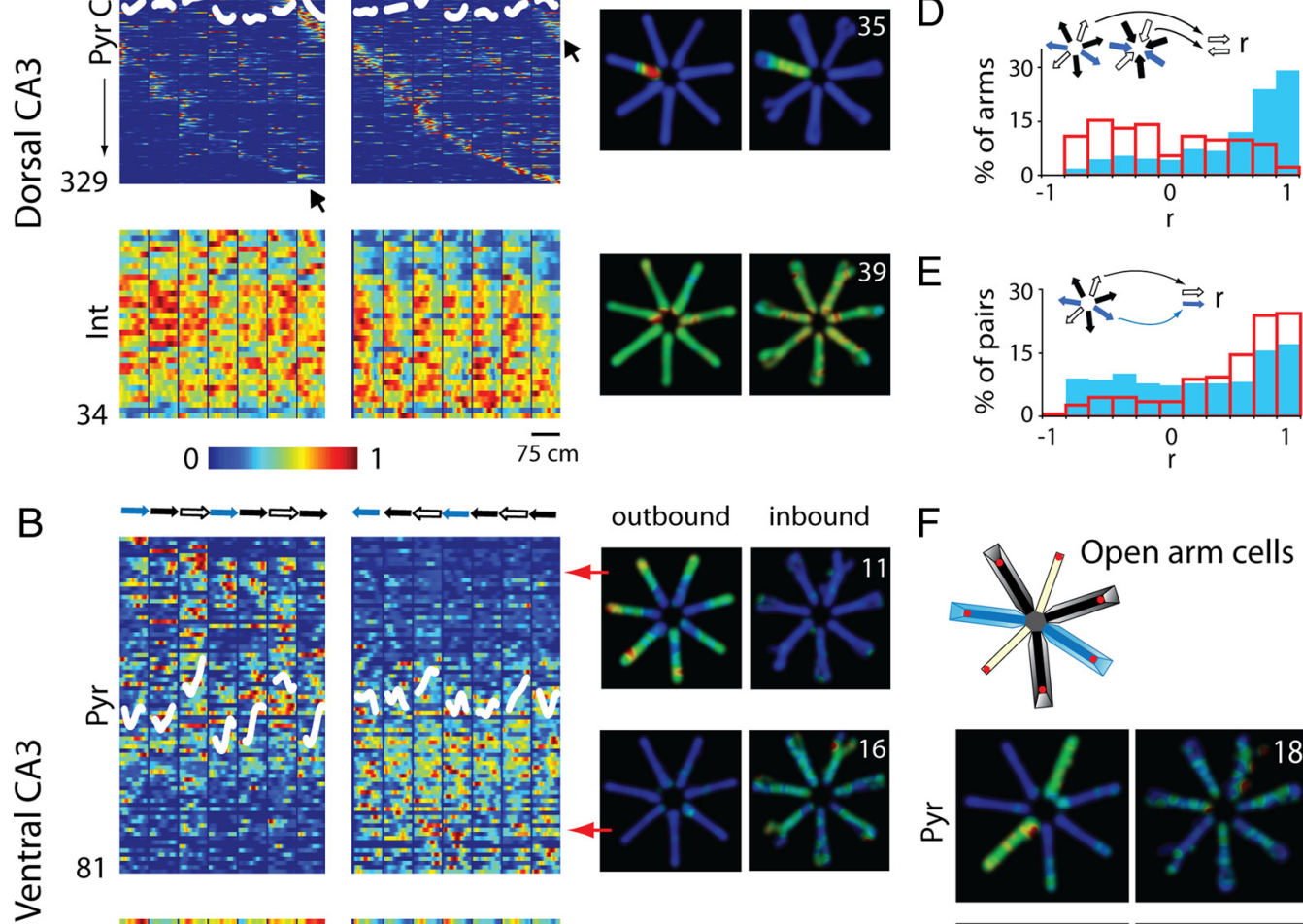

outbound

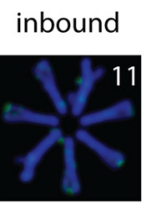

$\mathrm{F}$
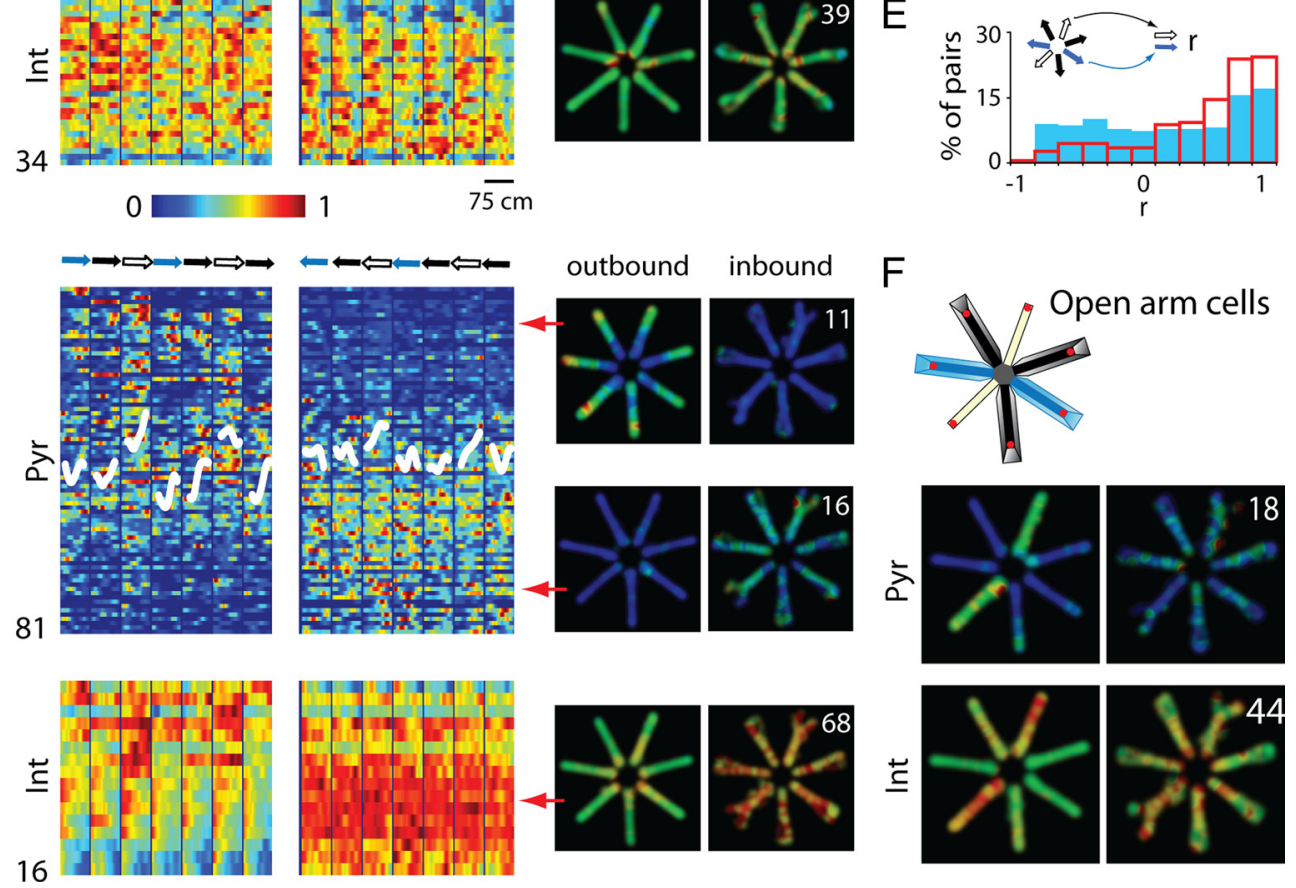

Figure 3. Differential representation of the radial maze in the dCA3 and vCA3 regions. A, Color-coded, normalized firing rates of dCA3 pyramidal cells (Pyr) and putative interneurons (Int) in each arm (horizontal arrows) during outbound and inbound travels (left and right panels, respectively). The arms of the radial maze were linearized and concatenated. Each line represents a single cell (1-329 pyramidal cells and 1-34 interneurons, respectively). Neurons are ordered according to the position of their firing fields in the maze. White line, Mean neuronal activity ("population rate"). Illustrative examples are shown on the right. Note that most dCA3 pyramidal cells are active in only one arm (diagonal) and also show an increase of firing at the same place fields in the opposite direction of travel (faint diagonal indicated by black arrows). B, Similar display for vCA3 neurons. Note that vCA3 neurons are typically active on multiple arms but do not repeat the firing fields in the opposite direction of travel. Note also the population rate increase in the open arms during outbound travels (white line). C, Distribution of arm representation of pyramidal cells. Definition of "active arm": arm with peak rate $>40 \%$ cell's peak rate. $\boldsymbol{D}$, Correlation between inbound and outbound firing patterns. The bottom axis label $r$ is the correlation coefficient. $\boldsymbol{E}$, Correlation between firing patterns of neurons in the different active arms. The bottom axis label $r$ is the correlation coefficient. $\boldsymbol{F}$, Examples of a vCA3 pyramidal cell and interneuron that differentiated between open and closed arms selectively during the outbound direction of travel.

stability was defined as the pixel-by-pixel correlation coefficient (see Fig. $2 D$, bottom axis label " $r$ ") between the firing rate maps of the first and second halves of the recording session.

Spatial information content. The spatial information content (Markus et al., 1994) was calculated according to the following equation:

$$
\text { Information per spike }=\sum_{i} P_{i} \cdot \frac{R_{i}}{R} \cdot \log _{2} \frac{R_{i}}{R},
$$

where $i$ is the pixel number, $P_{i}$ is the probability of occupancy of pixel $i, R_{i}$ is the mean firing rate in pixel $i$, and $R$ is the overall mean firing rate.

One-dimensional firing field vectors. The two-dimensional position of the rat was projected along the axes of the arms of the mazes. Each "linearized" arm of the radial and zigzag maze was divided into 100 equal pixels (50 pixels for the corner arms of the zigzag maze), and the number of spikes and occupancy times in each pixel were calculated. The resulting vectors of "spike count" and "occupancy time" were smoothed by convolving them with a Gaussian function (5 pixels half-width). The "firing field" vector was obtained from the ratio of spike count/occupancy time.

One-dimensional color-coded firing field plots. For each cell, the firing field vectors of all arms were concatenated into a single row. Next, the "row vectors" for all cells were vertically stacked into a single matrix. The row order was sorted according to their center of mass value (i.e., a vector position in which the integrals on each side are equal). For the radial maze, the outbound and inbound travel directions were concatenated and the center of mass was implemented on both travel directions together. As a result, cells that were mostly active during the outbound journey have lower center of mass values and are represented in the upper section of the plots (see Fig. $2 A, B$ ). For the zigzag maze, the "center of mass" was calculated over the leftward travels. The firing fields of ventral cells were smoothed using a larger Gaussian function (15 pixels half-width).

Arm firing selectivity in the radial maze. The Euclidian distance $(d)$ between arms firing rate vectors was computed for all possible pairs of 
arms. To estimate selective firing of neurons in the open arms relative to closed arms ("open arms" cells), the minimum over all possible values of $D=d$ (open|open) $-d$ (open|closed) was computed once (D1), and then recomputed for all possible permutations in arms order (D2-D22; 21 nonredundant permutations). A neuron was designated as an open arms cell if it met the significance criteria of D1 being distinct (nonoverlapping) from the distribution of D2-D22.

Spike autocorrelogram and theta indexes. Spike time autocorrelograms for all neurons were implemented with a time bin of $10 \mathrm{~ms}$, normalized to their maximum value between 100 and $150 \mathrm{~ms}$, clipped within $0-1$ range, and stacked into a single matrix. The cells were sorted according to the magnitude of theta modulation (see below). Each autocorrelogram was fit with the following equation:

$$
y(t)=[a(\sin (\omega t)+1)+b] \cdot e^{-|t| / \tau_{1}}+c \cdot e^{-t^{2} / \tau_{2}^{2}},
$$

where $t$ is the autocorrelograms time variable (from -700 to $700 \mathrm{~ms}$ ) and $a-c, \omega, t_{1}$, and $t_{2}$ are the fit parameters. The Gaussian term $c \cdot e^{-t^{2} / \tau_{2}{ }^{2}}$ was used to help fit the center peak of the autocorrelogram and its width was limited to a maximum value of $50 \mathrm{~ms}\left(\tau_{2}<50\right)$. Only autocorrelograms with at least 100 counts were included in this analysis. The theta indexes were defined as the ratio of the fit parameters $a / b$ (see Fig. 7; supplemental Fig. 6, available at www.jneurosci.org as supplemental material).

Phase precession calculation. Two methods were used to detect phase precession of spikes. In the first (direct) method, the theta phase of spikes is plotted as a function of the animal's position on the radial arm maze or zigzag maze (O'Keefe and Recce, 1993). Although this graphical method provides a measure of correlation strength between phase and position, it can quantify phase precession for neurons with high spatial coherence only. In our data set, only a minority of firing fields of vCA3 cells showed visually defined phase precession [similarly, in Kjelstrup et al. (2008), $<10 \%$ of vCA3 neurons showed reliable phase precession]. Because the direct method limited our analysis to "good" firing fields, we quantified phase precession by an indirect method. This method exploits the fact that for phase precession to be present, the neuron should oscillate faster than the reference LFP theta (O'Keefe and Recce, 1993; Maurer et al., 2005; Geisler et al., 2007). For this analysis, first the neuron's oscillation frequency was computed as $f_{n}=\omega / 2 \pi$, with $\omega$ being one of the parameters from the autocorrelogram fits. For the estimation of the theta frequency of the LFP, the peaks of theta waves were detected after bandpass filtering $(5-12 \mathrm{~Hz})$ the LFP, and a histogram of the interpeaks intervals was computed. The position of the histogram maximum was taken as the theta period $T$, and $f_{\mathrm{LFP}}=1 / T$, as the LFP theta frequency. Neurons were designated as "phase precessing" if the difference $\Delta f=f_{n}-f_{\text {LFP }}$ was $>0$ (Geisler et al., 2007).

\section{Results}

\section{Distinct representation by dorsal and ventral neurons}

We recorded simultaneously from both the dorsal and ventralmost parts of the hippocampus $(n=6$ rats) (Fig. $1 A, B)$ in rats foraging in three different testing apparatuses: open field, radial arm maze, and "zigzag" maze (Fig. $1 C-E$ ). Because of the curvature of the hippocampus, most recordings in the ventral part were performed in the CA3 region. Therefore, the following results focus on comparing dCA3 and vCA3 neuronal populations. Only neurons with well defined boundaries in the "cluster space" were included in the analyses (supplemental Fig. 1, available at www. jneurosci.org as supplemental material). Pyramidal cells and interneurons were separated by firing rate and spatial coverage criteria (dCA3 pyramidal cells, 1546; dCA3 interneurons, 107; vCA3 pyramidal cells, 351; vCA3 interneurons, 35) (supplemental Fig. 2, available at www.jneurosci.org as supplemental material). The session mean firing rates of putative pyramidal cells and interneurons in the two regions were comparable (supplemental Fig. $2 B$, available at www.jneurosci.org as supplemental material) (dCA3 pyramidal cells, $1.3 \pm 1.2 \mathrm{~Hz}$; vCA3 pyramidal cells, $1.5 \pm$
A Arms activity
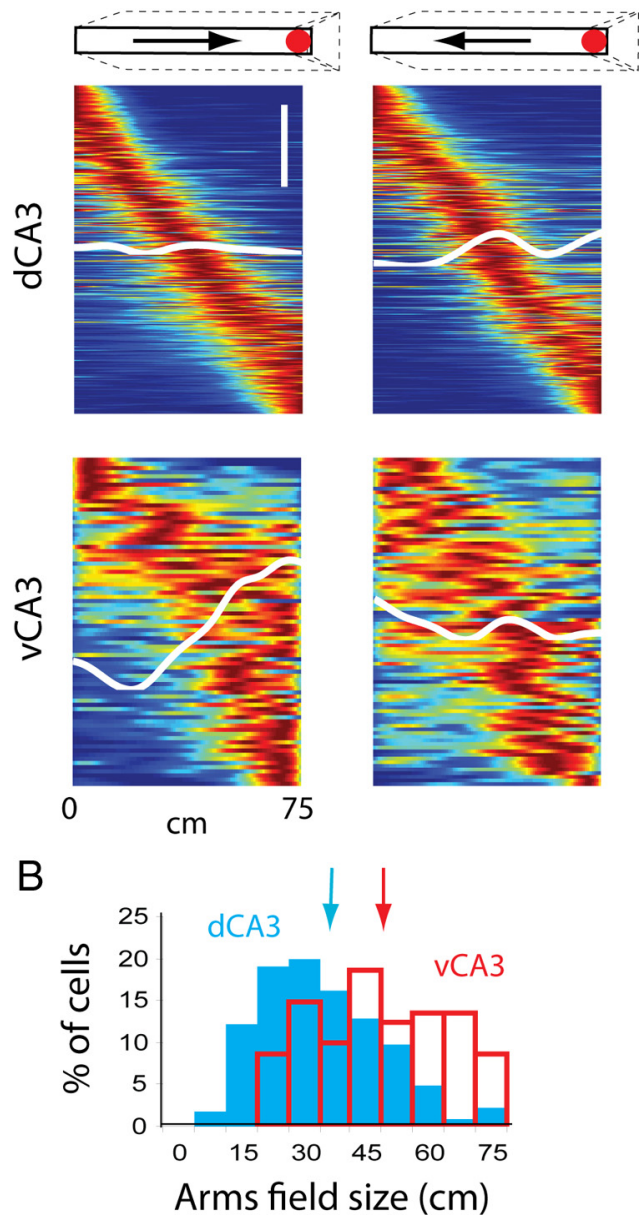

Figure 4. Single arm representation and field size. $A$, Activity fields of dCA3 and vCA3 neurons across arms, sorted by position of peak firing activity, shown separately during rewardbound (left) and center-bound (right) travels. White line, Sum of the neuronal activity (population rate). Note increasing activity of vCA3 neurons during outbound travel (67\% increase between first and last quarter; $p<0.01, t$ test). $\boldsymbol{B}$, Distribution of firing field sizes during reward-bound travel (values were virtually identical during center-bound travels). Note larger firing field sizes in vCA3 (arrow, mean).

$1.87 \mathrm{~Hz} ; p=0.11$; dCA3 interneurons, $32 \pm 21 \mathrm{~Hz}$; vCA3 interneurons, $34 \pm 19 \mathrm{~Hz} ; p=0.66$, unpaired $t$ test). Significantly fewer complex spike bursts ( $<6 \mathrm{~ms}$ interspike intervals) (Ranck, 1973) were present in vCA3 than in dCA3 pyramidal cells (supplemental Fig. $2 C$, available at www.jneurosci.org as supplemental material) ( $p<0.01$, unpaired $t$ test). In a small subset of experiments in which the same set of neurons could be reliably monitored in two or more testing environments, we found that neurons fired at different rates and at different positions or could be virtually silent in one environment while active in another one (supplemental Fig. 3, available at www.jneurosci.org as supplemental material) (Thompson and Best, 1989; Bostock et al., 1991) (cf. Muller, 1996).

Because place cell activity is typically assessed in two-dimensional environments (O'Keefe and Nadel, 1978; O'Keefe and Burgess, 1996), we first examined the discharge behavior of dorsal (569 pyramidal cells; 29 interneurons) and ventral cells (95 pyramidal cells; 6 interneurons) in the open field (Figs. 1C,2). As expected, dCA3 neurons had well defined single compact place fields in the open maze with high information content and spatial stability. In contrast, place fields with cone-shaped continuous 

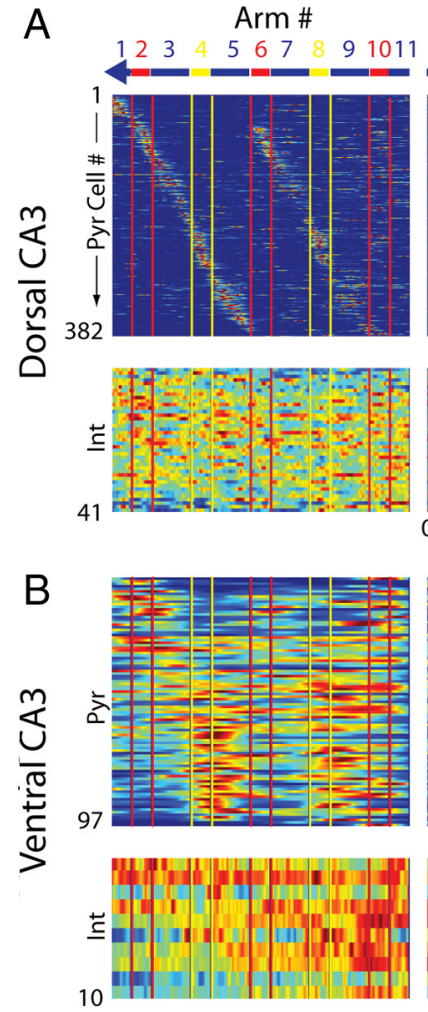
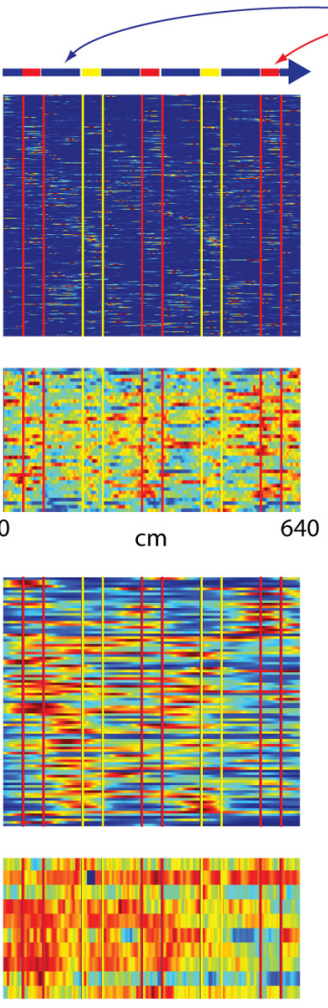

C

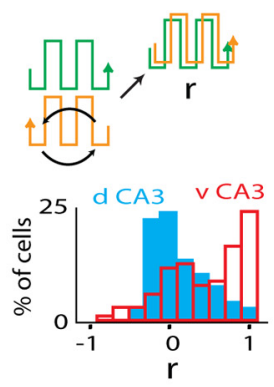

Field size
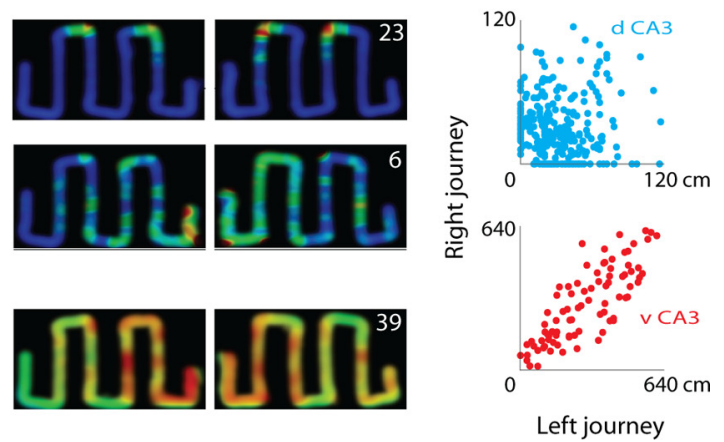

Figure 5. Differential representation of the zigzag maze in the dCA3 and vCA3 regions. $\boldsymbol{A}$, Color-coded, normalized firing rates of dCA3 pyramidal cells (Pyr) and interneurons (Int) in different segments of the maze. The arms of the maze (color-coded segments) were linearized and concatenated, and neurons were ordered according to their firing field position during leftward travels. Each horizontal line represents the same cell during leftward and rightward travels (left and right panels). The vertical lines separate respective corridors. Illustrative examples from each region are shown on the right. $\boldsymbol{B}$, Identical display for vCA3 neurons. Note that many vCA3 pyramidal neurons show mirrored firing patterns for opposite directions of travel. $\boldsymbol{C}$, Symmetry index (correlation between firing patterns on left travels and mirrored right travels). The bottom axis label $r$ is the correlation coefficient. $\boldsymbol{D}$, Field size of neurons during left versus right travels. Note the strong correlation between leftward and rightward field sizes for vCA3 neurons.

activity and surrounding silence, the defining criteria of place fields (O'Keefe and Nadel, 1978; O'Keefe and Burgess, 1996; Samsonovich and McNaughton, 1997), were only exceptionally observed in vCA3. Instead, vCA3 neurons fired at multiple locations with interruption of activity between them (Fig. $2 A$ ). We calculated each neuron's "spatial coverage" of the maze by two methods. First, we summed up all pixels in which the firing rate exceeded $20 \%$ of the peak firing rate and calculated the fraction of "active pixels" to all pixels. In the second method we created a contour line around the active pixels and calculated the percent of maze area it covered (Fig. $2 A$ ). Both methods indicated a significantly larger spatial coverage for vCA3 neurons (Fig. $2 B$ ) $(p<0.01$, unpaired $t$ test). In addition, vCA3 neurons showed significantly lower values of spatial information content (Fig. 2C) $(p<0.01$, unpaired $t$ test). Last, we examined the stability of firing patterns between the first and second halves of the recording sessions by calculating the correlation $(r)$ of firing rates in each pixel (Fig. 2D). We compared the stability of dorsal and ventral cells for groups of neurons with comparable field sizes. In all groups, the firing stability of vCA3 pyramidal cell population was significantly lower than that of dCA3 neurons (Fig. $2 D)(p<$ 0.01 , unpaired $t$ test). In summary, although position-related stability of firing patterns was present in a few vCA3 neurons, most of them lacked the defining criteria of place cells under our experimental conditions.

Next, we compared the firing properties of dorsal and ventral neurons in the radial arm maze (Fig. $1 D$ ) [pyramidal cells/interneurons: 329/34 (dorsal), 81/16 (ventral)]. As expected, dCA3 pyramidal cells typically had a single place field in only one of the seven arms of the radial maze (McNaughton et al., 1983) (Fig. $3 A, C)$. Several dCA3 neurons fired at the same arm location during the opposite direction journey, albeit at a lower rate (Fig. $3 A$, arrows pointing to the "ghost" diagonal pattern), resulting in a high pixel-by-pixel correlation between firing patterns of inbound and outbound journeys (Fig. 3D). Moreover, dCA3 neuron population evenly covered all visited locations, as shown by the steady population mean firing rates in the different arms (Fig. $3 A$, white line) and different portions of the arms (Fig. $4 A$, white line).

In contrast, (1) vCA3 neurons typically fired in multiple arms (Fig. $3 B, C$; supplemental Fig. 4, available at www.jneurosci.org as supplemental material) ( $p<0.01$, unpaired $t$ test) and showed stronger differentiation between inbound versus outbound journeys than dCA3 cells (Fig. 3D) $(p<0.01$, unpaired $t$ test). Importantly, the firing patterns were similar across different active arms for a given travel direction (inbound or outbound), as quantified by the high correlation of firing rates between arms (Fig. 3E) (vCA3 vs dCA3: $p<0.01$, unpaired $t$ test). (2) vCA3 population discriminated open from closed arms by showing a significant increase in population firing rate in the open arms (Fig. 3B, white line) (outbound, 34\%; $p<0.01$; inbound, $11 \%$; $p<0.01 ; t$ tests of open vs closed arms mean firing rates). Importantly, arm type differentiation occurred mainly during outbound direction of travel (Fig. 3F; supplemental Fig. 4, available at www.jneurosci.org as supplemental material), suggesting that the differential firing rates did not simply arise from the physical attributes of the arms but was affected by the context of the travel. (3) vCA3 population activity did not represent the arms length 
A

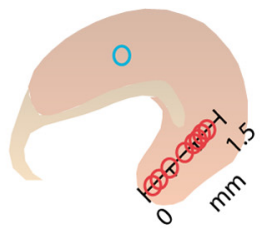

B

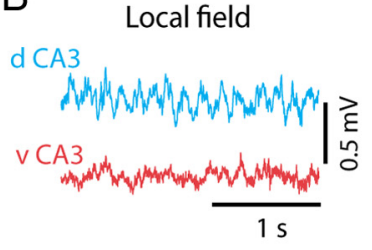

C

Power spectrum

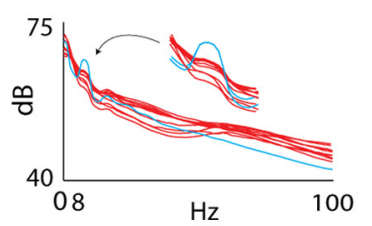

D
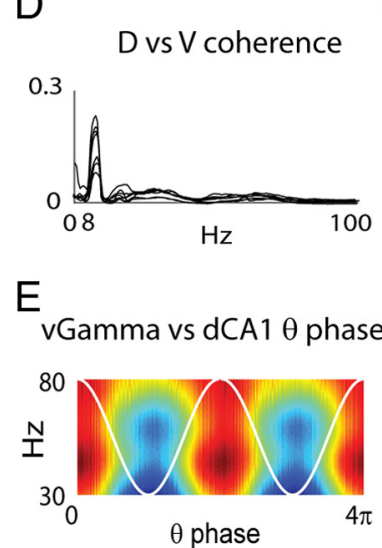

$\mathrm{F}$

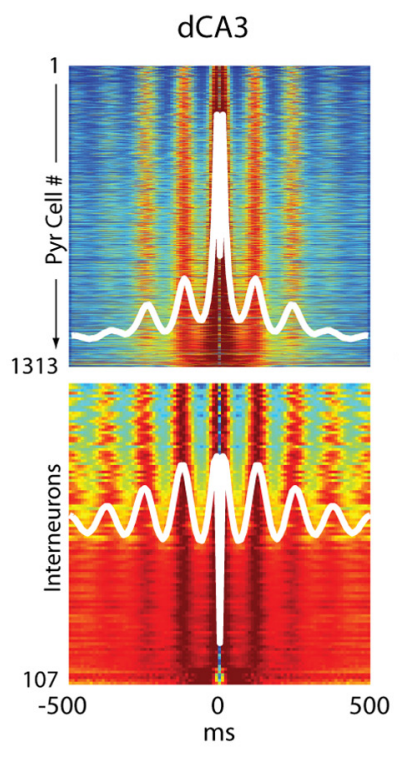

G

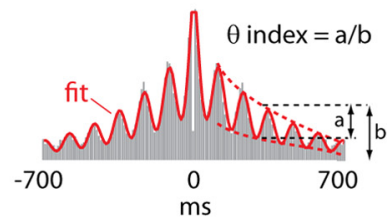

$\mathrm{H}$

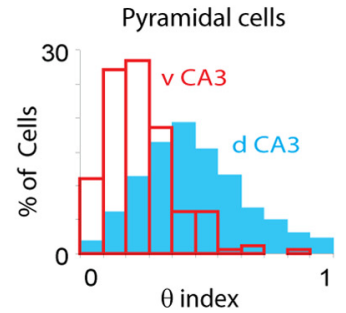

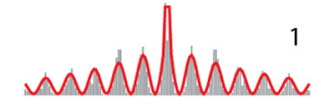

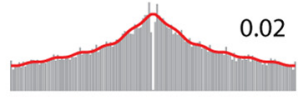

vCA3

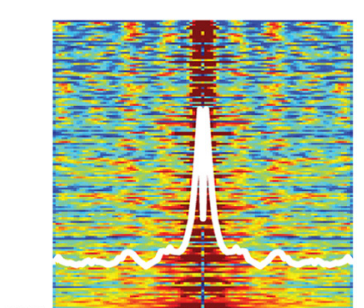

162

35

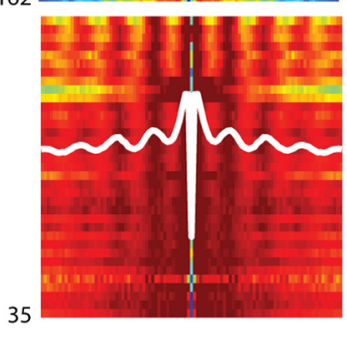

45

Interneurons

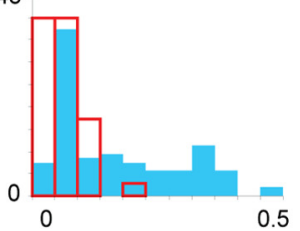

Figure 6. Theta oscillations in the dorsal and ventral hippocampus. $\boldsymbol{A}$, Recording sites in the ventral hippocampus. $\boldsymbol{B}$, LFP from the dorsal (dCA3) and ventral (vCA3) pyramidal layers. C, Power spectra of LFPs recorded from dCA3 (blue) and vCA3 (red) locations. $\boldsymbol{D}$, Coherence between dorsal and ventral LFP signals. $\boldsymbol{E}$, Phase modulation of gamma power in vCA3 pyramidal layer by theta LFP recorded in the dorsal hippocampus. $\boldsymbol{F}$, Color-coded autocorrelograms of pyramidal cells and interneurons, sorted by the magnitude of theta modulation strength (each line is a single neuron). White line, Population mean. G, Illustration of the calculation of theta index (see also supplemental Materials, available at www.jneurosci.org as supplemental material) and examples of neuron autocorrelograms with theta index of 1 and 0.02 . $\boldsymbol{H}$, Distribution of the theta index in dCA3 and vCA3 pyramidal cells and interneurons. Note low theta index values in the ventral population.

evenly but the firing rate steeply increased during outbound travels (Fig. 4A) (67\% increase between first and last quarter; $p<$ $0.01 ; t$ test of last quarter vs first quarter mean firing rates). The size of single-arm firing fields, quantified as the length of activity between the $20 \%$ boundaries of the peak firing rate in a given arm was $39 \%$ larger in vCA3 cells, although both regions showed large field size variability (Fig. $4 B)(p<0.01$, unpaired $t$ test).

To summarize, in contrast to dCA3 neurons, most vCA3 neurons discharged in multiple arms of the radial maze, were active in equivalent segments of the outbound journeys to the reward sites, and differentiated "open" versus "closed" arms. Putative vCA3 interneurons showed mostly similar patterns to vCA3 pyramidal cells (Fig. 3 B, F; supplemental Fig. 4, available at www. jneurosci.org as supplemental material).

Possible reasons for the similarity of vCA3 repeated firing patterns in the radial maze could be the similar geometry of the

arms or the presence of rewards at the end of each arm. To disambiguate these hypotheses, we designed a zigzag maze (Fig. $1 E$ ) [pyramidal cells/interneurons: $382 / 41$ (dorsal), 97/10 (ventral)], in which the rat had to run through two geometrically identical corridor configurations before reaching the reward. According to the first hypothesis, vCA3 neurons should repeat their firing at each repeated segment of the zigzag maze, whereas the second hypothesis predicts symmetric firing pattern between left and right journeys. Unexpectedly, several dCA3 pyramidal neurons fired repetitively at geometrically identical positions of the zigzag maze (note double diagonal firing patterns in Fig. 5A), reminiscent of the "path equivalence" firing patterns of entorhinal cortical neurons (Frank et al., 2000; Derdikman et al., 2009). vCA3 neurons also showed repeating firing patterns (Fig. $5 B$ ), suggesting that the local maze cues affected both dCA3 and vCA3 neurons. However, a large fraction of vCA3 but not dCA3 neurons fired in a symmetric manner during left and right journeys (Fig. $5 A$, $B$; supplemental Fig. 5, available at www.jneurosci. org as supplemental material), resulting in a high pixel-by-pixel correlation between the left and reversed-right journeys for vCA 3 cells but not dCA 3 cells (Fig. $5 C$; $p<0.01$, unpaired $t$ test). Consistent with the journey-symmetric firing pattern, the sizes of firing fields during left and right journeys were strongly correlated for vCA3 $(r=0.81)$ but not dCA3 $(r=-0.02)$ pyramidal cells (Fig. 5D). Symmetric firing rate patterns were also pronounced in the vCA3 interneuron population (Fig. 5B). In summary, local cues in the zigzag maze modulated both dCA3 and vCA3 neuronal activity. However, only vCA3 neurons discharged symmetric firing patterns during left and right journeys, indicating that for at least a fraction of ventral neurons the direction of travel toward the reward location or other factors had more influence than either the local cues in the maze or the extramaze landmarks in the room.

\section{Reduced theta modulation of neurons in the ventral hippocampus}

Because spatial properties of dorsal hippocampal place cells are strongly linked to hippocampal theta oscillations (Huxter et al., 2003; O'Keefe and Burgess, 2005), we examined the temporal firing properties of neurons and their relationship to LFPs in the dorsal and ventral regions. In contrast to the highly rhythmic pattern of LFP in the dorsal CA3 pyramidal layer, theta rhythmicity in vCA3 was often hard to detect visually even when continuous theta oscillations were present in the dorsal hippocampus (Fig. 6A). Potentially because of the intermittent nature of vCA3 theta oscillations, theta power was significantly lower in the ventral compared with the dorsal hippocampus (Fig. 6C) (56 $\pm 11 \%$ 

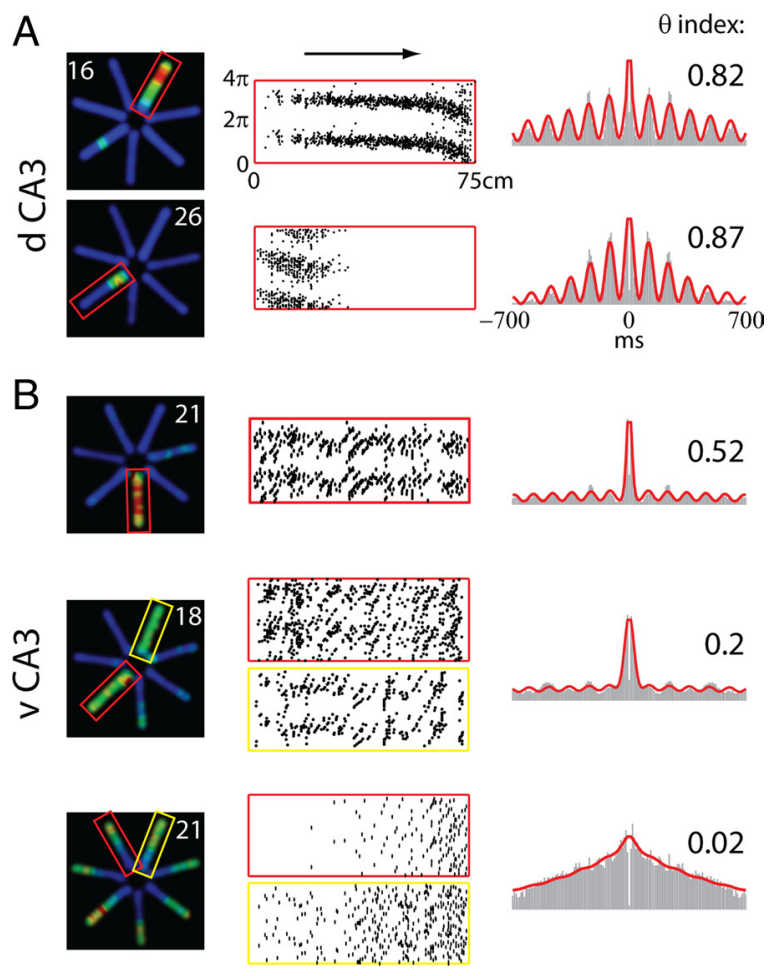

C

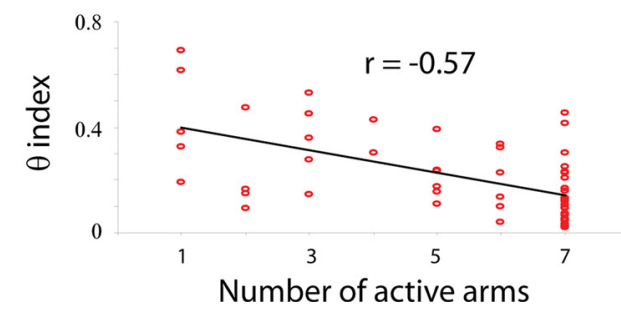

Figure 7. Parallel decrease of spatial precision and theta oscillations. $\boldsymbol{A}, \boldsymbol{B}$, Firing field, theta phase of spikes as a function of rat's position, and autocorrelogram of example dCA3 $(\boldsymbol{A})$ and $\mathrm{vCA} 3(\boldsymbol{B})$ neurons. Theta indices are indicated next to the autocorrelograms. Note that theta modulation or phase precession of spikes is not visible in cells with low theta indices. $C$, Inverse relationship between theta index and the numbers of arms in which the neurons were active.

reduction of $8 \mathrm{~Hz}$ power band; mean $\pm \mathrm{SD} ; p<0.01$, unpaired $t$ test). However, when present, vCA3 theta was temporally coordinated with theta derived from the dorsal hippocampus, as shown by both the distinct coherence peak in the theta band (Fig. $6 D$ ) and the significant phase modulation of ventral gamma power by dorsal theta oscillations (Fig. $6 E)(19 \pm 12 \%$; $p<0.01, t$ test).

In addition to LFP theta changes, we also examined the firing properties of single pyramidal cells and interneurons. Whereas dCA3 place cells and interneurons showed strongly rhythmic spike autocorrelograms at theta frequency (O'Keefe and Recce, 1993; Csicsvari et al., 1999; Geisler et al., 2007), rhythmicity of vCA3 pyramidal cells and interneurons was markedly less pronounced (Fig. 6F). To quantify the magnitude of unit theta rhythmicity, we fit a sinusoidal function to each spike autocorrelogram and used the relative amplitude of the sinusoid component as an index of theta rhythmicity (Fig. 6G) (see Materials and Methods). Compared with $85 \%$ of dCA3 pyramidal cells (interneurons, $37 \%$ ), only $25 \%$ of vCA3 principal cells (interneurons, $3 \%$ ) had a theta index $>0.2$ (Fig. $6 H$ ).

Interestingly, a significant inverse relationship between theta index and the number of radial maze arms in which the neuron was active could be observed within vCA3 population (Fig. $7 B, C$ ) $(r=-0.57)$. This finding indicates that the more the firing patterns of individual vCA3 neurons resembled the dCA3 place fields, the more strongly they were modulated by theta, supporting the hypothesis of a strong link between theta rhythmicity and the spatial aspect of hippocampal computation. Finally, although the fraction of theta-rhythmic neurons in vCA3 was robustly lower than in dCA3, these theta-rhythmic vCA3 neurons also showed phase precession (Fig. 7 A,B; supplemental Fig. 6, available at www.jneurosci.org as supplemental material).

\section{Discussion}

Our findings demonstrate hitherto unknown differences between the dorsal and the ventral-most parts of the hippocampus in both environmental representation and temporal dynamics. We hypothesize that, as spatial information become less precise and theta rhythms fade in the septo-temporal direction, other types of cortical information gain stronger representation toward the temporal end of the hippocampus.

\section{Septo-temporal shift of representations in the hippocampus} Our study supports and extends previous observation in the intermediate and ventral hippocampus (Jung et al., 1994; Poucet et al., 1994; Maurer et al., 2005; Kjelstrup et al., 2008). vCA3 pyramidal cells displayed position-dependent firing in both the radial arm and zigzag mazes, with firing fields larger than those of dCA3 cells, supporting the hypothesis that neurons at different septotemporal levels simultaneously represent the same environment with different spatial resolution (Jung et al., 1994; Maurer et al., 2005; McNaughton et al., 2006; Kjelstrup et al., 2008; Moser et al., 2008). The progressively coarser representation of space toward the ventral hippocampus is in register with the growing size of "grids" and the decreasing frequency of the theta oscillations of the neurons along the dorso-ventral axis of the medial entorhinal cortex (Hafting et al., 2005; Giocomo et al., 2007; Moser et al., 2008).

However, several of our observations cannot be explained by an increase of spatial scale alone, since size expansion of place fields could not explain numerous novel features of vCA3 firing patterns in our testing environments. (1) In contrast to dCA3, continuous, cone-shaped place fields, the defining characteristic of place cells in two-dimensional environments (O'Keefe and Nadel, 1978; Samsonovich and McNaughton, 1997), were exceptionally rare in vCA3. (2) In the radial maze, vCA3 neurons differentiated the inbound and outbound directions of travel more effectively than dCA3 cells, discharged similar patterns in different arms, and discriminated open from close arms. (3) vCA3, but not dCA3, cells discharged mirror-symmetric firing patterns in the zigzag maze.

Our findings suggest that, in addition to the expanding field sizes, the septo-temporal differences result from an increasing representation of nonspatial information in the ventral hippocampus. Several findings indicate that vCA3 cells are more sensitive to local maze cues than extramaze landmarks (Olton et al., 1979; Thompson and Best, 1989). vCA3 neurons fired at physically similar segments of the arms of the radial maze and differentiated between open and closed arms. In the zigzag maze, the firing rates of vCA3 neurons varied in the different corridors. However, the physical features of local maze cues cannot fully account for the differences between dCA3 and vCA3 neurons, since vCA3 neurons discharged differentially during inbound and outbound journeys in the radial maze and a subset of cells showed mirror-symmetric firing in the zigzag maze. In addition to the role of local cues, we suggest that nonspatial factors that 
affect the firing patterns of ventral hippocampal neurons may include reward and emotional features. In support of this hypothesis, vCA3 firing patterns were more strongly controlled by the reward locations than distal room cues: (1) The lack of coneshaped place fields in the open arena would be expected from such a reward location-defined reference since the rewards were scattered all over the arena in that task. (2) The multiple and similar-size firing fields in the radial maze may be explained by the presence of rewards in all arms. The strong differentiation between inbound and outbound (i.e., reward location-bound) travels by vCA3 cells in the radial maze can also be explained by the polarizing role of the reward or goal. (3) The mirrorsymmetric firing patterns of a subset of vCA3 cells in the zigzag maze also support the hypothesis of goal directed bias of firing patterns. The role of emotional factors in affecting firing patterns in the ventral hippocampus is supported by the differential firing patterns of vCA3 neurons in the open and closed arms of the radial arm maze. Providing more direct evidence for these hypotheses will require manipulating reward locations and emotional valences in future experiments.

The known anatomical connections of the hippocampus may offer some clues to the physiological findings in our study. The ventral part of the medial entorhinal cortex, which conveys information to the ventral hippocampus in the rat, receives only very sparse representation from visual and other sensory areas, modalities thought to be critical for place cells (O'Keefe and Nadel, 1978). Neurons in this region show poor spatial modulation (Quirk et al., 1992; Frank et al., 2000; Hargreaves et al., 2005). However, inputs from the amygdala and hypothalamus, two structures involved in emotion and reward representation (Hikosaka et al., 2008), reach only the ventral quadrant of the rodent hippocampus (Witter et al., 1989; Pitkanen et al., 2000; Petrovich et al., 2001). From the above perspective, a possible explanation for the ability of vCA3 neurons to discriminate open from closed arms of the radial maze is that such differentiation reflects amygdala-mediated emotional modulation of neuronal activity in the ventral hippocampus (Paré et al., 2002). This hypothesis is in line with lesion experiments showing the lack of ventral hippocampal contribution to spatial learning but its stronger involvement in emotional mediation (Moser et al., 1995; Kjelstrup et al., 2002; Pentkowski et al., 2006; Bast et al., 2009) and the observed increase of theta power in the open arms in $5 \mathrm{HT}_{1 \mathrm{~A}}$-deficient mice (Gordon et al., 2005). Finally, the subpopulation of vCA3 neurons, which accelerated spiking activity during reward-bound travels may correspond to the hypothesized "goal" cells (O’Keefe and Burgess, 1996) and/or "headdirection accumulator" cells (Kubie and Fenton, 2009), whose postulated function is to integrate travel distance.

\section{Temporal dynamics in the dorsal and ventral hippocampus}

In agreement with the study of Kjelstrup et al. (2008), theta rhythmic cells showed a slower rate of phase precession in ventral hippocampus than in dorsal hippocampus. However, an unexpected finding of the present experiments was the strong reduction of theta LFP power and the small fraction of theta-rhythmic neurons in the ventral hippocampus. These findings further illustrate the deterioration of precise spatial representation toward the temporal end of the hippocampus, because theta oscillations have been suggested to be an obligatory aspect of place cell activity (O'Keefe and Recce, 1993; O’Keefe and Burgess, 2005; Geisler et al., 2007; Jeewajee et al., 2008).

The mechanisms that account for the dorsal versus ventral differences in theta dynamics have yet to be disclosed, but there are several candidates. First, whereas the ventral quadrant of the hippocampus receives its cholinergic inputs from the horizontal limb of the diagonal band (Amaral and Lavenex, 2007) and through a ventral pathway (Gage et al., 1983), the rest of the hippocampus is innervated by neurons of the vertical limb and medial septum (Stewart and Fox, 1990). Second, the intrinsic resonant properties of ventral hippocampal cells may also be different, a possibility supported by the expression of distinct ion channel genes in the temporal quarter of the hippocampus (Lein et al., 2007). Third, the parent cells of the entorhinal afferents to the different septo-temporal parts of the hippocampus may possess different rhythmic properties (Giocomo et al., 2007). Finally, parvalbumin-containing interneurons in ventral hippocampus are significantly fewer than in the septal part (Seress and Ribak, 1983).

The paucity of theta LFP and theta rhythmic neurons in the ventral hippocampus of the rat may be relevant to the often reported paucity of theta oscillations in the primate hippocampus in waking (Arnolds et al., 1980; Kahana et al., 2001; Ekstrom et al., 2005) as well as during REM sleep (Cantero et al., 2003). With the development of the neocortex and the caudalward shift of the hippocampus (Amaral and Lavenex, 2007), the ventral quadrant of the rodent hippocampus expanded disproportionally to become the uncus and corpus of the primate hippocampus (Fig. $1 A$ ), whereas growth of the dorsal part (to become the posterior tail) was less expressed. This assumption is supported by the pattern of inputs from the hypothalamus and amygdala, confined to the ventral tip of the hippocampus in rodents (Witter et al., 1989; Pitkanen et al., 2000; Petrovich et al., 2001) but present throughout the uncus and body of the monkey hippocampus (Saunders et al., 1988). In line with this reasoning, spatial modulation of hippocampal neurons was observed only in the more caudal (uncal) part of the monkey hippocampus (Colombo et al., 1998). Combining these findings with the present observations, we hypothesize that theta-rhythmic firing and theta field oscillations are more prominent in the tail part of the primate hippocampus.

Despite reduced in power, theta oscillations in ventral hippocampus were coherent with dorsal theta oscillations, as hypothesized recently by Lubenov and Siapas (2009). Such temporal coordination, also expressed by the phase modulation of ventral CA3 units and gamma power by dorsal theta oscillations, may be brought about by phase-coherent inputs from the medial septum (Stewart and Fox, 1990), the longitudinally extending CA3 axons ( $\mathrm{Li}$ et al., 1994) or extensive collaterals of the "longrange" family of interneurons (Jinno et al., 2007). In conclusion, our findings suggest a shift from spatial to progressively nonspatial representation along the septo-temporal axis. Despite the reduction of theta oscillations in the ventral hippocampus, our findings also suggest that the theta rhythm may serve as a mechanism to temporally bind spatial and nonspatial representations in the different segments of the hippocampus.

\section{References}

Amaral D, Lavenex P (2007) Hippocampal neuroanatomy. In: The hippocampus book (Andersen P, Morris R, Amaral D, Bliss T, O’Keefe J, eds). Oxford: Oxford UP.

Andersen P, Bliss VP, Skrede KK (1971) Lamellar organization of the hippocampal excitatory pathways. Exp Brain Res 13:222-238.

Arnolds DE, Lopes da Silva FH, Aitink JW, Kamp A, Boeijinga P (1980) The spectral properties of hippocampal EEG related to behaviour in man. Electroencephalogr Clin Neurophysiol 50:324-328.

Ashton D, Van Reempts J, Haseldonckx M, Willems R (1989) Dorsalventral gradient in vulnerability of CA1 hippocampus to ischemia: a combined histological and electrophysiological study. Brain Res 487:368-372. 
Bannerman DM, Grubb M, Deacon RM, Yee BK, Feldon J, Rawlins JN (2003) Ventral hippocampal lesions affect anxiety but not spatial learning. Behav Brain Res 139:197-213.

Bast T, Wilson IA, Witter MP, Morris RG (2009) From rapid place learning to behavioral performance: a key role for the intermediate hippocampus. PLoS Biol 7:730-746.

Bostock E, Muller RU, Kubie JL (1991) Experience-dependent modifications of hippocampal place cell firing. Hippocampus 1:193-205.

Bragdon AC, Taylor DM, Wilson WA (1986) Potassium-induced epileptiform activity in area CA3 varies markedly along the septotemporal axis of the rat hippocampus. Brain Res 378:169-173.

Cantero JL, Atienza M, Stickgold R, Kahana MJ, Madsen JR, Kocsis B (2003) Sleep-dependent theta oscillations in the human hippocampus and neocortex. J Neurosci 23:10897-10903.

Colombo M, Fernandez T, Nakamura K, Gross CG (1998) Functional differentiation along the anterior-posterior axis of the hippocampus in monkeys. J Neurophysiol 80:1002-1005.

Csicsvari J, Hirase H, Czurko A, Buzsáki G (1998) Reliability and state dependence of pyramidal cell-interneuron synapses in the hippocampus: an ensemble approach in the behaving rat. Neuron 21:179-189.

Csicsvari J, Hirase H, Czurkó A, Mamiya A, Buzsáki G (1999) Oscillatory coupling of hippocampal pyramidal cells and interneurons in the behaving rat. J Neurosci 19:274-287.

Deadwyler SA, Bunn T, Hampson RE (1996) Hippocampal ensemble activity during spatial delayed-nonmatch-to-sample performance in rats. J Neurosci 16:354-372.

Derdikman D, Whitlock JR, Tsao A, Fyhn M, Hafting T, Moser MB, Moser EI (2009) Fragmentation of grid cell maps in a multicompartment environment. Nat Neurosci 12:1325-1332.

Dolorfo CL, Amaral DG (1998) Entorhinal cortex of the rat: topographic organization of the cells of origin of the perforant path projection to the dentate gyrus. J Comp Neurol 398:49-82.

Ekstrom AD, Caplan JB, Ho E, Shattuck K, Fried I, Kahana MJ (2005) Human hippocampal theta activity during virtual navigation. Hippocampus 15:881-889.

Ferbinteanu J, Shapiro ML (2003) Prospective and retrospective memory coding in the hippocampus. Neuron 40:1227-1239.

Frank LM, Brown EN, Wilson M (2000) Trajectory encoding in the hippocampus and entorhinal cortex. Neuron 27:169-178.

Freund TF, Buzsaki G (1996) Interneurons of the hippocampus. Hippocampus 6:347-470.

Gage FH, Björklund A, Stenevi U (1983) Reinnervation of the partially deafferented hippocampus by compensatory collateral sprouting from spared cholinergic and noradrenergic afferents. Brain Res 268:27-37.

Geisler C, Robbe D, Zugaro M, Sirota A, Buzsáki G (2007) Hippocampal place cell assemblies are speed-controlled oscillators. Proc Natl Acad Sci U S A 104:8149-8154.

Giocomo LM, Zilli EA, Fransén E, Hasselmo ME (2007) Temporal frequency of subthreshold oscillations scales with entorhinal grid cell field spacing. Science 315:1719-1722.

Gordon JA, Lacefield CO, Kentros CG, Hen R (2005) State-dependent alterations in hippocampal oscillations in serotonin 1A receptor-deficient mice. J Neurosci 25:6509-6519.

Hafting T, Fyhn M, Molden S, Moser MB, Moser EI (2005) Microstructure of a spatial map in the entorhinal cortex. Nature 436:801-806.

Hampson RE, Simeral JD, Deadwyler SA (1999) Distribution of spatial and nonspatial information in dorsal hippocampus. Nature 402:610-614.

Hargreaves EL, Rao G, Lee I, Knierim JJ (2005) Major dissociation between medial and lateral entorhinal input to dorsal hippocampus. Science 308:1792-1794.

Harris KD, Henze DA, Csicsvari J, Hirase H, Buzsáki G (2000) Accuracy of tetrode spike separation as determined by simultaneous intracellular and extracellular measurements. J Neurophysiol 84:401-414.

Hazan L, Zugaro M, Buzsáki G (2006) (2006) Klusters, NeuroScope, NDManager: a free software suite for neurophysiological data processing and visualization. J Neurosci Methods 155:207-216.

Hikosaka O, Bromberg-Martin E, Hong S, Matsumoto M (2008) New insights on the subcortical representation of reward. Curr Opin Neurobiol 18:203-208.

Huxter J, Burgess N, O'Keefe J (2003) Independent rate and temporal coding in hippocampal pyramidal cells. Nature 425:828-832.

Jeewajee A, Barry C, O’Keefe J, Burgess N (2008) Grid cells and theta as oscillatory interference: electrophysiological data from freely moving rats. Hippocampus 18:1175-1185.

Jinno S, Klausberger T, Marton LF, Dalezios Y, Roberts JD, Fuentealba P, Bushong EA, Henze D, Buzsáki G, Somogyi P (2007) Neuronal diversity in GABAergic long-range projections from the hippocampus. J Neurosci 27:8790-8804.

Johnson A, Redish AD (2007) Neural ensembles in CA3 transiently encode paths forward of the animal at a decision point. J Neurosci 27:1217612189.

Jung MW, Wiener SI, McNaughton BL (1994) Comparison of spatial firing characteristics of units in dorsal and ventral hippocampus of the rat. J Neurosci 14:7347-7356.

Kahana MJ, Seelig D, Madsen JR (2001) Theta returns. Curr Opin Neurobiol 11:739-744.

Kjelstrup KB, Solstad T, Brun VH, Hafting T, Leutgeb S, Witter MP, Moser EI, Moser MB (2008) Finite scale of spatial representation in the hippocampus. Science 321:140-143.

Kjelstrup KG, Tuvnes FA, Steffenach HA, Murison R, Moser EI, Moser MB (2002) Reduced fear expression after lesions of the ventral hippocampus. Proc Natl Acad Sci U S A 99:10825-10830.

Kubie JL, Fenton AA (2009) Heading-vector navigation based on headdirection cells and path integration. Hippocampus 19:456-479.

Lein ES, Hawrylycz MJ, Ao N, Ayres M, Bensinger A, Bernard A, Boe AF, Boguski MS, Brockway KS, Byrnes EJ, Chen L, Chen L, Chen TM, Chin MC, Chong J, Crook BE, Czaplinska A, Dang CN, Datta S, Dee NR, et al. (2007) Genome-wide atlas of gene expression in the adult mouse brain. Nature 445:168-176.

Li XG, Somogyi P, Ylinen A, Buzsáki G (1994) The hippocampal CA3 network: an in vivo intracellular study. J Comp Neurol 339:181-208.

Lieb JP, Engel J Jr, Gevins A, Crandal PH (1981) Surface and deep EEG correlates of surgical outcome in temporal lobe epilepsy. Epilepsia 22:515-538.

Lubenov EV, Siapas G (2009) Hippocampal theta oscillations are travelling waves. Nature 459:534-539.

Maggio N, Segal M (2007) Striking variations in corticosteroid modulation of long-term potentiation along the septotemporal axis of the hippocampus. J Neurosci 27:5757-5765

Markus EJ, Barnes CA, McNaughton BL, Gladden VL, Skaggs WE (1994) Spatial information content and reliability of hippocampal CA1 neurons: effects of visual input. Hippocampus 4:410-421.

Maurer AP, Vanrhoads SR, Sutherland GR, Lipa P, McNaughton BL (2005) Self-motion and the origin of differential spatial scaling along the septotemporal axis of the hippocampus. Hippocampus 15:841-852.

McNaughton BL, Barnes CA, O'Keefe J (1983) The contributions of position, direction, and velocity to single unit activity in the hippocampus of freely-moving rats. Exp Brain Res 52:41-49.

McNaughton BL, Battaglia FP, Jensen O, Moser EI, Moser MB (2006) Path integration and the neural basis of the "cognitive map." Nat Rev Neurosci 7:663-678

Moser EI, Kropff E, Moser MB (2008) Place cells, grid cells, and the brain's spatial representation system. Annu Rev Neurosci 31:69-89.

Moser MB, Moser EI (1998) Functional differentiation in the hippocampus. Hippocampus 8:608-619.

Moser MB, Moser EI, Forrest E, Andersen P, Morris RG (1995) Spatial learning with a minislab in the dorsal hippocampus. Proc Natl Acad Sci U S A 92:9697-9701.

Muller RU (1996) A quarter of a century of place cells. Neuron 17:813-822.

O'Keefe J, Burgess N (1996) Geometric determinants of the place fields of hippocampal neurones. Nature 381:425-428.

O'Keefe J, Burgess N (2005) Dual phase and rate coding in hippocampal place cells: theoretical significance and relationship to entorhinal grid cells. Hippocampus 15:858-866.

O'Keefe J, Nadel L (1978) The hippocampus as a cognitive map. Oxford: Oxford UP.

O’Keefe J, Recce ML (1993) Phase relationship between hippocampal place units and the EEG theta rhythm. Hippocampus 3:317-330.

Olton DS, Becker JT, Handelmann GE (1979) Hippocampus, space, and memory. Behav Brain Sci 2:313-365.

Paré D, Collins DR, Pelletier JG (2002) Amygdala oscillations and the consolidation of emotional memories. Trends Cogn Sci 6:306-314.

Pastalkova E, Itskov V, Amarasingham A, Buzsáki G (2008) Internally gen- 
erated cell assembly sequences in the rat hippocampus. Science 321:1322-1327.

Pentkowski NS, Blanchard DC, Lever C, Litvin Y, Blanchard RJ (2006) Effects of lesions to the dorsal and ventral hippocampus on defensive behaviors in rats. Eur J Neurosci 23:2185-2196.

Petrovich GD, Canteras NS, Swanson LW (2001) Combinatorial amygdalar inuts to hippocampal domains and hypothalamic behavior systems. Brain Res Brain Res Rev 38:247-289.

Pitkanen A, Pikkarainen M, Nurminen N, Ylinen A (2000) Reciprocal connections between the amygdala and the hippocampal formation, perirhinal cortex, and postrhinal cortex in rat. Ann N Y Acad Sci 911:369-391.

Poucet B, Thinus-Blanc C, Muller RU (1994) Place cells in the ventral hippocampus of rats. Neuroreport 5:2045-2048.

Quirk GJ, Muller RU, Kubie JL, Ranck JB Jr (1992) The positional firing properties of medial entorhinal neurons: description and comparison with hippocampal place cells. J Neurosci 12:1945-1963.

Ranck JB Jr (1973) Studies on single neurons in dorsal hippocampal formation and septum in unrestrained rats. I. Behavioral correlates and firing repertoires. Exp Neurol 41:461-531.

Rempel-Clower NL, Zola SM, Squire LR, Amaral DG (1996) Impaired recognition memory in patients with lesions limited to the hippocampal formation. J Neurosci 16:5233-5255.

Risold PY, Swanson LW (1996) Structural evidence for functional domains in the rat hippocampus. Science 272:1484-1486.

Samsonovich A, McNaughton BL (1997) Path integration and cognitive mapping in a continuous attractor neural network model. J Neurosci 17:5900-5920.

Saunders RC, Rosene DL, Van Hoesen GW (1988) Comparison of the efferents of the amygdala and the hippocampal formation in the rhesus monkey: II. Reciprocal and non-reciprocal connections. J Comp Neurol 271:185-207.
Seress L, Ribak CE (1983) GABAergic cells in the dentate gyrus appear to be local circuit and projection neurons. Exp Brain Res 50:173-182.

Small SA (2002) The longitudinal axis of the hippocampal formation: its anatomy, circuitry, and role in cognitive function. Rev Neurosci 13: 183-194.

Stewart M, Fox SE (1990) Do septal neurons pace the hippocampal theta rhythm? Trends Neurosci 13:163-168.

Thompson LT, Best PJ (1989) Place cells and silent cells in the hippocampus of freely-behaving rats. J Neurosci 9:2382-2390.

Van Hoesen GW, Pandya DN (1975) Some connections of the entorhinal (area 28) and perirhinal (area 35) cortices of the rhesus monkey. III. Efferent connections. Brain Res 95:39-59.

Verney C, Baulac M, Berger B, Alvarez C, Vigny A, Helle KB (1985) Morphological evidence for a dopaminergic terminal field in the hippocampal formation of young and adult rat. Neuroscience 14:1039-1052.

Verwer RW, Meijer RJ, Van Uum HF, Witter MP (1997) Collateral projections from the rat hippocampal formation to the lateral and medial prefrontal cortex. Hippocampus 7:397-402.

Wible CG, Findling RL, Shapiro M, Lang EJ, Crane S, Olton DS (1986) Mnemonic correlates of unit activity in the hippocampus. Brain Res 399:97-110.

Witter M, Amaral DG (2004) Hippocampal formation. In: The rat nervous system, Ed 3 (Paxinos G, ed). San Diego: Elsevier Academic.

Witter MP, Groenewegen HJ, Lopes da Silva FH, Lohman AH (1989) Functional organization of the extrinsic and intrinsic circuitry of the parahippocampal region. Prog Neurobiol 33:161-253.

Wittner L, Henze DA, Záborszky L, Buzsáki G (2007) Three-dimensional reconstruction of the axon arbor of a CA3 pyramidal cell recorded and filled in vivo. Brain Struct Funct 212:75-83.

Wood ER, Dudchenko PA, Robitsek RJ, Eichenbaum H (2000) Hippocampal neurons encode information about different types of memory episodes occurring in the same location. Neuron 27:623-633. 